\title{
Dynamics of free surface perturbations along an annular viscous film
}

\author{
Linda B. Smolka* \\ Department of Mathematics, Bucknell University, Lewisburg, Pennsylvania 17837, USA \\ Justin North \\ Department of Physics, Ohio State University, Columbus, Ohio 43210-1117, USA \\ Bree K. Guerra \\ Department of Physics, University of Texas at Austin, Austin, Texas 78712-0264, USA
}

(Received 4 July 2007; revised manuscript received 24 December 2007; published 3 March 2008)

\begin{abstract}
It is known that the free surface of an axisymmetric viscous film flowing down the outside of a thin vertical fiber under the influence of gravity becomes unstable to interfacial perturbations. We present an experimental study using fluids with different densities, surface tensions, and viscosities to investigate the growth and dynamics of these interfacial perturbations and to test the assumptions made by previous authors. We find that the initial perturbation growth is exponential, followed by a slower phase as the amplitude and wavelength saturate in size. Measurements of the perturbation growth for experiments conducted at low and moderate Reynolds numbers are compared to theoretical predictions developed from linear stability theory. Excellent agreement is found between predictions from a long-wave Stokes flow model [Craster and Matar, J. Fluid Mech. 553, 85 (2006)] and data, while fair to excellent agreement (depending on fiber size) is found between predictions from a moderate-Reynolds-number model [Sisoev et al., Chem. Eng. Sci. 61, 7279 (2006)] and data. Furthermore, we find that a known transition in the longer-time perturbation dynamics from unsteady to steady behavior at a critical flow rate $Q_{c}$ is correlated with a transition in the rate at which perturbations naturally form along the fiber. For $Q<Q_{c}$ (steady case), the rate of perturbation formation is constant. As a result, the position along the fiber where perturbations form is nearly fixed, and the spacing between consecutive perturbations remains constant as they travel $2 \mathrm{~m}$ down the fiber. For $Q>Q_{c}$ (unsteady case), the rate of perturbation formation is modulated. As a result, the position along the fiber where perturbations form oscillates irregularly, and the initial speed and spacing between perturbations varies, resulting in the coalescence of neighboring perturbations further down the fiber.
\end{abstract}

DOI: 10.1103/PhysRevE.77.036301

PACS number(s): 47.20.Dr, 47.55.df, 47.85.mb

\section{INTRODUCTION}

Coatings are commonly applied to the exterior of thin cylindrical wires or fibers to provide protection and/or enhance performance (e.g., electrical wire and fiber-optic cable). Methods of coating include extruding a fiber through a die (die coating) or drawing a fiber from a liquid bath (dip coating) [1-5]. During the coating process, a uniform liquid film can become unstable to interfacial perturbations that may develop further into droplets [6,7]. This effect, which detracts from the quality of a coating, has inspired a wide array of studies on the formation and motion of perturbations on cylindrical fibers [6-13].

Fibers can also be coated by a continuously fed axisymmetric fluid flow down the length of a vertical fiber (see Fig. $1)$, as has been examined in several analytical and experimental studies [14-19]. The geometry of the unperturbed flow is an annular film with a fixed internal boundary and a free surface at the outer fluid-air interface. It is well known that the free surface of this annular film becomes unstable to interfacial perturbations, as shown in Fig. 1. Herein we present an experimental study on an annular viscous film with a particular focus on the initial formation and longer-

\footnotetext{
*1smolka@bucknell.edu
}

time dynamics of perturbations along the film free surface.

A related problem to annular films is that of the motion of cylindrical jets, which in contrast have no fixed internal boundary. Analytical studies on the motion and stability of inviscid and viscous jets date back to the work of Plateau [20], Lord Rayleigh [21,22], Weber [23], and Chandrasekhar [24]. It is known that capillary effects drive perturbation growth along the jet free surface; this is often referred to as the Plateau-Rayleigh instability. Predictions for perturbation growth along inviscid and viscous jets, developed from temporal linear stability theory [24], were tested in experiments by Donnelly and Glaberson [25]. They found strong agreement between the theoretical and measured dispersion relations for an inviscid jet and fair agreement for a viscous jet. The dynamics of free surface perturbations along cylindrical

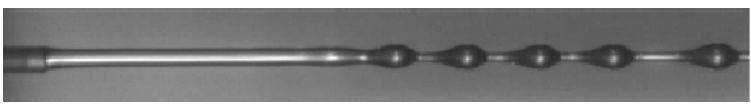

FIG. 1. An annular film of viscous fluid (80:20 glycerol:water solution) flowing down the outside of a thin nylon fiber; the surrounding fluid is air. Note that the figure has been rotated by $90^{\circ}$ with gravity acting toward the right. Perturbations develop along the free surface some distance down the fiber; once formed, these perturbations continue to travel down the fiber. Image length $=9.7 \mathrm{~cm}$. 
jets and annular films are, however, quite different. In the cylindrical case, jet breakup occurs when the perturbations become sufficiently large [25], while in the annular case, the large-amplitude perturbations remain connected by a liquid film $[14,15,19]$.

Recently, several theoretical studies have analyzed the temporal linear stability of an annular viscous film flowing down a vertical fiber in the Stokes $[14,15]$ and moderateReynolds-number $[17,18]$ limits; the base flow in these studies is assumed to be a steady, unidirectional, parallel flow. Here we test these results by determining whether (i) the base flow used in $[14,15,17,18]$ matches the experimental flow; and (ii) the dispersion relations derived in the Stokes and moderate-Reynolds-number limits $[15,18]$ correctly predict the nascent growth of perturbations measured in lowand moderate-Reynolds-number flows.

As free surface perturbations travel down a vertical fiber, many interesting phenomena occur $[14,15,18,19]$. In experiments, Kliakhandler, Davis, and Bankoff (KDB) observed three types of behavior far down the length of the fiber $(\approx 2 \mathrm{~m})[14]$. At the highest flow rate (regime $a)$, the film between perturbations is thick and uniform, and fastermoving perturbations collide into slower-moving perturbations (unsteady behavior). At an intermediate flow rate (regime $b$ ), the spacing, size, and speed of the perturbations are constant so that no collisions occur (steady behavior). And, at the lowest flow rate (regime $c$ ), the fluid periodically drips from the tank (rather than jets, as in regimes $a$ and $b$ ), which creates a regular spacing between perturbations near the tank outlet. The long time between drips allows the film connecting consecutive perturbations to thin and subsequently become unstable to smaller capillary perturbations. (Figure 1 in [14] illustrates these three regimes of behavior.) Simulations of a Stokes flow model developed by KDB qualitatively captured two of the three observed behaviors (regimes $b$ and $c$ ), while the behavior associated with the highest flow rate (regime $a$ ) could not be replicated [14].

Craster and Matar (CM) [15] select a different scaling than KDB to derive an evolution equation for the free surface. Using traveling wave solutions, their Stokes flow model quantitatively predicted the perturbation speed and height of regime $a$ measured by KDB. The model also qualitatively captured regime $c$, though the steady pattern of perturbation spacing found in regime $b$ could not be matched with traveling wave solutions. In experiments, $\mathrm{CM}$ observed regime $b$ near the tank outlet; however, they found that the regularly spaced pattern of perturbations disassembled itself further down the fiber. From this observation, CM concluded that regime $b$ is a transient rather than a steady regime [15].

The contradiction between observations of regime $b$ (steady behavior) by $\mathrm{KDB}$ and $\mathrm{CM}$ motivated us to look more closely at the steady and unsteady states by examining the dynamics of the perturbations where they initially form along the fiber. In our experiments, we find that the occurrence of regime $b$ is dependent on the fiber size. Using fluids with different densities, surface tensions, and viscosities and a moderate-sized fiber we observe regimes $a$ (unsteady), $b$ (steady), and $c$ (dripping), whereas with a thinner fiber we observe only regimes $a$ and $c$. In the former case, we find that the flow transitions abruptly from unsteady to steady behavior at a critical flow rate $Q_{c}$ (the value of $Q_{c}$ is dependent on the particular fluid) [26]. In a recent independent study, Duprat et al. [19] explain the transition from regime $a$ (unsteady behavior) to regime $b$ (steady behavior) as a transition from convective to absolute instability. In their experiments with silicone oil using a range of fiber and orifice radii, they find that the transition occurs only at intermediate film thicknesses and for sufficiently small fiber radii; at thin or thick film thickness, they find that the perturbation behavior remains convective (unsteady) [19]. Here we find that the transition from unsteady to steady behavior is also correlated with the rate at which perturbations naturally form along the fiber. For $Q<Q_{c}$ (steady case), the rate of perturbation formation is constant. As a result, the position along the fiber where perturbations form is nearly fixed, and the spacing between consecutive perturbations remains constant as they travel $2 \mathrm{~m}$ down the fiber. For $Q>Q_{c}$ (unsteady case), the rate of perturbation formation is modulated. As a result, the position along the fiber where perturbations form oscillates irregularly, and the initial speed and spacing between perturbations vary, resulting in the coalescence of neighboring perturbations further down the fiber.

The paper is organized as follows. The experimental setup and properties of the unperturbed flow are presented in Sec. II. Measurements of the perturbation growth are compared to analytical predictions for Stokes and moderate-Reynoldsnumber conditions in Sec. III. The perturbation behavior exhibited in regimes $a$ (unsteady) and $b$ (steady) near the tank outlet are closely examined in Sec. IV. Conclusions are provided in Sec. V.

\section{EXPERIMENTAL SETUP AND DETAILS}

\section{A. Experimental apparatus}

The experimental setup consists of viscous fluids, a reservoir tank-orifice assembly, nylon fishing line, a high-speed digital imaging camera, illumination, a computer, and edgedetection software [a schematic of the experiment is shown in Fig. 2(a)]. The reservoir tank (6 1 capacity) is graduated at $100 \mathrm{ml}$ increments to measure flow rate. An orifice, machined with a flat edge (inner radius $=0.11 \mathrm{~cm}$, outer radius $=0.16 \mathrm{~cm}$, length $=2.6 \mathrm{~cm}$ ), is attached to the bottom of the tank to ensure a reproducible solid/fluid/air contact line in the experiment. A nylon fiber (radius $=0.010$ or 0.029 $\mathrm{cm}$ ) anchored from above, passes through the center of the tank-orifice assembly, and is held vertically plumb with weights attached $2 \mathrm{~m}$ below the orifice. The fluid, which is gravitationally forced from the tank, coats the fiber to create an annular film. To reduce air currents and other noise during data collection, the entire apparatus is enclosed by an aluminum frame with plastic sheet sidewalls and top.

The motion of the annular film is recorded using a highspeed digital imaging camera (Phantom v4.2) at rates between 1000 and 4000 frames/s and an image size of 64 $\times 512$ pixels $^{2}$ with the camera focused on approximately the upper $10 \mathrm{~cm}$ of the fiber. Illumination is obtained using silhouette photography following [27], with a $250 \mathrm{~W}$ lamp, an experimental grade one-way transparent mirror (Edmund Scientific, A40,047), and high-contrast reflective screen ma- 
(a)

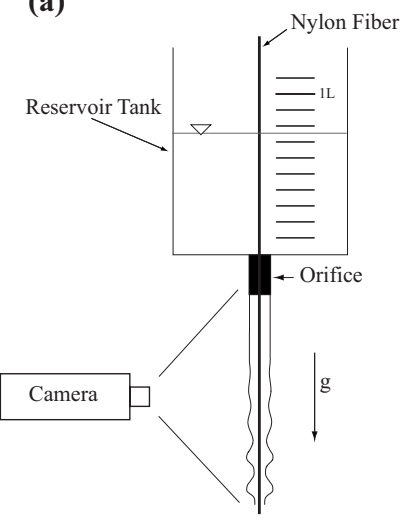

(b)

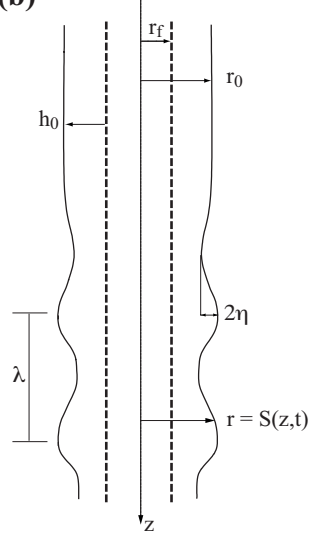

FIG. 2. (a) Schematic of experimental setup. (b) Cross section of an annular film flowing down the outside of a fiber of radius $r_{f}$ (not to scale). The unperturbed film radius measured from the fiber centerline is $r_{0}$, unperturbed film thickness $h_{0}$, perturbation amplitude $\eta(z, t)$, and perturbation wavelength $\lambda$.

terial (Scotchlite 3M 7615). Movies of the annular film are recorded and downloaded to a computer using camera software. The free surface of the film is determined from movie images using an edge-detection algorithm. The algorithm locates the free surface by interpolating the maxima positions in a gradient image; the gradient image is produced using the Frei-Chen operator [28]. The algorithm can detect the edge of the film free surface to within approximately $1 / 10$ of a pixel, which for the screen resolution in our experiments corresponds to $\approx 0.002 \mathrm{~cm}$.

The experimental fluids consist of castor oil, vegetable oil (Crisco), and an 80:20 glycerol:water solution (by weight). The temperature, density $(\rho)$, surface tension $(\gamma)$, and dynamic viscosity $(\eta)$ of the fluids, and the fiber radius $\left(r_{f}\right)$, framing rate, and screen resolution used in the experiments are listed in Table I. Experiments with castor oil and vegetable oil were conducted with the thicker fiber $\left(r_{f}=0.029 \mathrm{~cm}\right)$, while experiments with glycerol solution were conducted with both sized fibers. The surface tension was measured at room temperature using a Fisher 21 tensiomat and viscosity was measured using a temperaturecontrolled cone and plate rheometer (Brookfield, Model DV$\mathrm{III}+$ ). The fluid temperature varied by less than $4.6 \%, 1.9 \%$, and $1.4 \%$ in the castor oil, vegetable oil, and glycerol solu-

tion experiments, respectively. We note that (i) the selection of fluids allows us to independently probe the influence of surface tension or viscosity on flow behavior (with $r_{f}$ $=0.029 \mathrm{~cm}$ ) since castor oil and vegetable oil have comparable surface tensions, while vegetable oil and the glycerol solution have comparable viscosities; and (ii) the glycerol solution experiments allow us to probe the influence of fiber radius on the flow behavior.

\section{B. Base flow properties}

In each experiment, the reservoir tank drained under the influence of gravity and the elapsed time and tank volume were recorded as the fluid passed each $100 \mathrm{ml}$ mark to determine the flow rate $(Q)$. Data were collected only while the flow was jetting from the orifice and an unperturbed region of the film free surface was present near the orifice (corresponding to regimes $a$ and $b$ [14]). Measurements of the flow rate during each experimental run for vegetable oil (squares), glycerol solution (triangles), and castor oil (circles) are shown in Fig. 3(a); open symbols correspond to $r_{f}$ $=0.010 \mathrm{~cm}$, filled symbols to $r_{f}=0.029 \mathrm{~cm}$. (Note that in each experiment the flow rate decreases as the tank volume decreases.) Figure 3(a) shows that the flow rate increases linearly over the range of tank volume used in each experimental run. Furthermore, the flow rate (i) decreases with increasing fiber radii (glycerol solution); and (ii) decreases with increasing viscosity $\left(Q_{\text {castor }}\right.$ is an order of magnitude less than $Q_{\text {glycerol }}$ and $Q_{\text {vegetable }}$ ) and increases with increasing surface tension $\left(Q_{\text {glycerol }}>Q_{\text {vegetable }}\right)$ for fixed $r_{f}$.

The unperturbed film radius $r_{0}$, measured from the fiber centerline to the unperturbed free surface [see Fig. 2(b)], was measured using edge-detection software. Figure 3(b) shows that the unperturbed radius increases with flow rate in each experimental run. This trend is similar to behavior observed in dip coating, in which the film thickness increases with the velocity at which the fiber is drawn from the fluid source at sufficiently low withdrawal rates [1-3,5]. Comparing data for the glycerol solution (filled triangles) and vegetable oil (squares) experiments, we find that at a fixed flow rate higher surface tension (glycerol solution) results in a thinner unperturbed film. Furthermore, comparing glycerol solution experiments, we find that at a fixed flow rate an increase in the fiber radii results in a thicker unperturbed film. In our experiments the films are thick, with the ratio of unperturbed film

TABLE I. Fluid properties and experimental conditions.

\begin{tabular}{|c|c|c|c|c|c|c|}
\hline Fluid & $\begin{array}{c}\text { Temperature } \\
\left({ }^{\circ} \mathrm{C}\right)\end{array}$ & $\begin{array}{c}\rho \\
\left(\mathrm{g} / \mathrm{cm}^{3}\right)\end{array}$ & $\begin{array}{c}\gamma \\
(\mathrm{dyn} / \mathrm{cm})\end{array}$ & $\begin{array}{c}\mu \\
(\mathrm{g} / \mathrm{cm} \mathrm{s})\end{array}$ & $\begin{array}{c}\text { Framing rate } \\
\text { (frames/s) }\end{array}$ & $\begin{array}{l}\text { Screen resolution } \\
(\mathrm{cm} / \mathrm{pixel})\end{array}$ \\
\hline Castor oil $^{\mathrm{a}}$ & 21.9 & 0.94 & 36.8 & 8.48 & 1000 & 0.0190 \\
\hline Vegetable oil $^{\mathrm{a}}$ & 21.6 & 0.92 & 34.3 & 0.58 & 4000 & 0.0180 \\
\hline Glycerol solution $^{\mathrm{a}}$ & 21.2 & 1.21 & 60.4 & 0.54 & 2000 & 0.0186 \\
\hline Glycerol solution $^{\mathrm{b}}$ & 21.7 & 1.21 & 60.4 & 0.52 & 2000 & 0.0189 \\
\hline
\end{tabular}



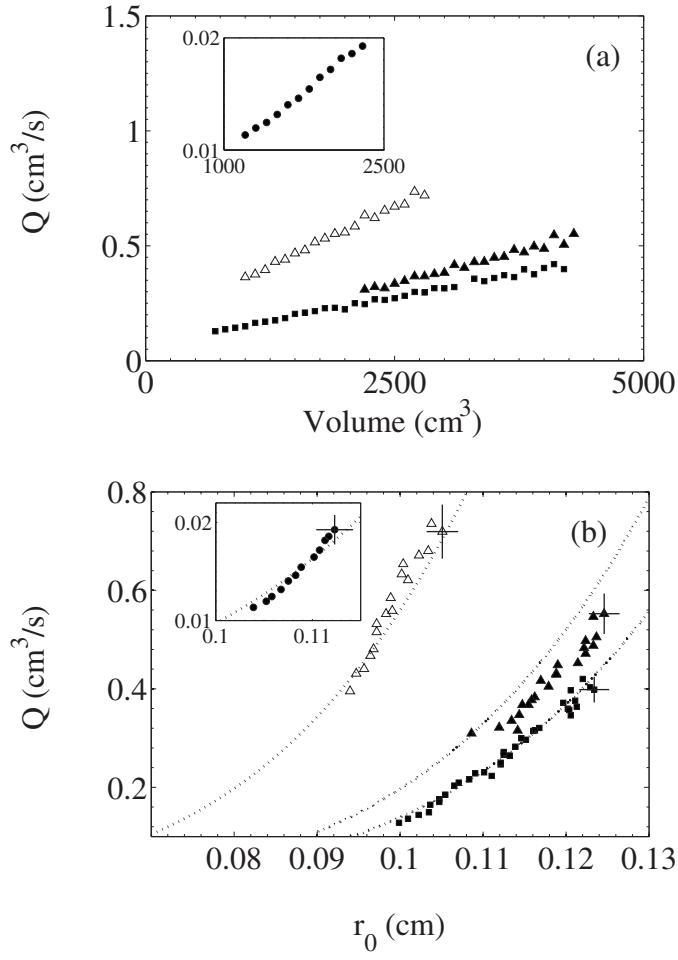

FIG. 3. (a) Experimental flow rate as a function of the reservoir tank volume. (b) Flow rate as a function of the unperturbed film radius: experimental data (symbols), theory given by Eq. (4) (dotted line); bars represent the resolution of experimental measurements. Symbols correspond to vegetable oil (squares), glycerol solution (triangles), castor oil (circles), open symbols $\left(r_{f}=0.010 \mathrm{~cm}\right)$, and filled symbols $\left(r_{f}=0.029 \mathrm{~cm}\right)$.

thickness to fiber radius in the range $2.5<h_{0} / r_{f}<9.5$.

The relevant forces that characterize the dynamics of an annular film can be determined by considering two dimensionless groups, the Reynolds number and Bond number. Following CM [15], we define characteristic length and velocity scales as

$$
\mathcal{L}=\gamma /\left(\rho g r_{0}\right), \quad \mathcal{V}=\rho g r_{0}^{2} / \mu,
$$

where $g$ is the gravitational constant of acceleration, the Reynolds number as $\operatorname{Re}=\rho \mathcal{V} L / \mu$, which compares inertial to viscous effects, and the Bond number as $\mathrm{Bo}=\rho g r_{0}^{2} / \gamma$, which compares gravitational to surface tension effects [15]. The values of Re and Bo for our experiments, listed in Table II,

TABLE II. The Reynolds number and Bond number measured in experiments.

\begin{tabular}{ccc}
\hline \hline Fluid & Re & Bo \\
\hline Castor oil $^{\mathrm{a}}$ & $0.049-0.054$ & $0.26-0.32$ \\
Vegetable oil $^{\mathrm{a}}$ & $9.4-11.6$ & $0.26-0.40$ \\
Glycerol solution $^{\mathrm{a}}$ & $27.2-31.2$ & $0.23-0.30$ \\
Glycerol solution $^{\mathrm{b}}$ & $24.9-28.4$ & $0.17-0.22$ \\
\hline${ }^{\mathrm{a}}{ }_{r_{f}=0.029 \mathrm{~cm} .}$ & \\
${ }^{\mathrm{b}}{ }_{r_{f}}=0.010 \mathrm{~cm}$. & &
\end{tabular}

indicate that inertial effects are negligible for castor oil $\left[\operatorname{Re} \sim O\left(10^{-2}\right)\right]$, in contrast to the vegetable oil and glycerol solution experiments $[\operatorname{Re} \sim O(10)]$, and surface tension dominates over gravitational effects in the formation of interfacial perturbations for all three fluids $\left[\mathrm{Bo} \sim O\left(10^{-1}\right)\right]$.

Experimental $[14,15]$ and theoretical $[14,15,17,18]$ studies often assume that the base flow of an annular film is steady, unidirectional, and parallel. Under these conditions, the unperturbed flow with free surface located at $r=r_{0}$ and constant pressure field $p(r, z, t)=p_{0}$ is described by the boundary value problem for the axial velocity $w(r)$,

$$
\mu r^{-1} \partial_{r}\left(r \partial_{r} w\right)+\rho g=0, \quad w\left(r_{f}\right)=0, \quad \partial_{r} w\left(r_{0}\right)=0,
$$

where the boundary conditions include no-slip at the fiber and zero tangential stress at the free surface. Equations (2) can be solved exactly for the axial velocity to obtain

$$
w(r)=\frac{\rho g}{4 \mu}\left[2 r_{0}^{2} \ln \left(r / r_{f}\right)+r_{f}^{2}-r^{2}\right] .
$$

Using (3), the flow rate of an annular film can be expressed in terms of $r_{f}$ and $r_{0}$ :

$$
\begin{aligned}
Q\left(r_{f}, r_{0}\right)= & 2 \pi \int_{r_{f}}^{r_{0}} r w(r) d r=\frac{\rho g \pi}{8 \mu}\left[4 r_{f}^{2} r_{0}^{2}+4 r_{0}^{4} \ln \left(r_{0} / r_{f}\right)-r_{f}^{4}\right. \\
& \left.-3 r_{0}^{4}\right] .
\end{aligned}
$$

It is worth noting that the functional forms of the axial velocity and flow rate used in the theoretical studies of KDB [14], CM [15], and Sisoev et al. [18] are equivalent to (3) and (4), respectively. Next, we compare (4) to experimental data to test the assumption that the base flow of an annular film is steady, unidirectional, and parallel.

Figure 3(b) shows a comparison of the flow rate as a function of the unperturbed film radius for vegetable oil, glycerol solution, and castor oil measured directly in experiments (symbols) and using (4) (dotted lines). We find excellent agreement between (4) and the experimental data for castor oil (circles), vegetable oil (squares), and glycerol solution with $r_{f}=0.010 \mathrm{~cm}$ (open triangles), which indicates that the flow in these experiments is well approximated by a steady, unidirectional, parallel flow. In the glycerol solution experiment with $r_{f}=0.029 \mathrm{~cm}$ (filled triangles), the theory overestimates $27 \%$ of the experimental data by as much as $4.5 \%$; the fiber was slightly off center in these experiments (shown in Fig. 1), which may account for this discrepancy. Based on the strong agreement in Fig. 3(b), we find that (3) and (4) are valid for the flow conditions in our experiments, $\mathrm{Bo} \ll 1$ and $\mathrm{Re} \lesssim 30$. The experiments conducted by KDB [14] and CM [15] meet this criterion; thus we conclude that their assumption that the flow is steady, unidirectional, and parallel is indeed valid.

In the following sections, we examine the dynamics of a perturbed annular film including the initial formation and longer-time dynamics of interfacial perturbations along the free surface. 


\section{PERTURBATION GROWTH}

The image in Fig. 1 illustrates the capillary instability an annular viscous film undergoes as the unperturbed free surface becomes unstable to undulations that develop into largeamplitude perturbations. Next we present experimental observations on the growth of these interfacial perturbations and compare their initial growth to theoretical predictions developed from linear stability analysis. Before proceeding, we first recount relevant stability results developed in the Stokes [15] and moderate-Reynolds-number flow [18] limits.

\section{A. Linear stability results}

\section{Stokes flow}

Craster and Matar [15] derive a long-wave Stokes flow evolution equation for the free surface of the annular film, $r=S(z, t)$, under the assumptions that the unperturbed film radius $r_{0}$ is small relative to the capillary length $l_{c}=\gamma / \rho g r_{0}$ (i.e., $\left.\mathrm{Bo}=r_{0} / l_{c}=\rho g r_{0}^{2} / \gamma \ll 1\right)$ and the Reynolds number is sufficiently small $[\operatorname{Re} \leq O(1)]$, to obtain

$$
\begin{aligned}
\partial_{\hat{t}}\left(\hat{S}^{2}\right) & +\frac{1}{8} \partial_{\hat{z}}\left\{[ \partial _ { \hat { z } } ( \frac { 1 } { \hat { S } } - ( \mathrm { Bo } ) ^ { 2 } \partial _ { \hat { z } \hat { z } } \hat { S } ) - 1 ] \left[\alpha^{4}-4 \hat{S}^{2} \alpha^{2}+3 \hat{S}^{4}\right.\right. \\
& \left.\left.-4 \hat{S}^{4} \ln (\hat{S} / \alpha)\right]\right\}=0
\end{aligned}
$$

where $\hat{S}, \hat{z}$, and $\hat{t}$ are dimensionless variables satisfying the scalings

$$
S=r_{0} \hat{S}, \quad z=\mathcal{L} \hat{z}, \quad t=\mathcal{L} \hat{t} / \mathcal{V},
$$

and $\alpha=r_{f} / r_{0}$. Conducting a linear stability analysis by perturbing about the base flow

$$
\hat{S}(\hat{z}, \hat{t})=1+\hat{S}_{1} e^{(i m \hat{z}+\sigma \hat{t})},
$$

where $m$ is the (real) dimensionless wavenumber and $\sigma$ is the (complex) dimensionless growth rate, CM obtain the following dispersion relation for the growth rate:

$$
\begin{aligned}
\sigma= & \frac{m^{2}}{16}\left(\mathrm{Bo}^{2} m^{2}-1\right)\left[\alpha^{4}-4 \alpha^{2}+3+4 \ln (\alpha)\right]-\frac{i m}{2}\left[\alpha^{2}-1\right. \\
& -2 \ln (\alpha)] .
\end{aligned}
$$

\section{Moderate-Reynolds-number flow}

In an analytical study, Trifonov [17] derived model equations for fluid flowing down the inside or outside of a vertical cylinder at moderate Reynolds number; the model includes evolution equations for the film thickness $h(z, t)=S(z, t)-r_{f}$ and volumetric flow rate $q(z, t)$. In a recent study, Sisoev et al. [18] rescale Trifonov's equations for flow down the outside of a vertical cylinder, casting the model in terms of a generalized falling film model [29] (see Eqs. (11)-(13) in [18]). Conducting a linear stability analysis of the rescaled equations by perturbing about the base solution

$$
\hat{h}(\hat{z}, \hat{t})=1+\hat{h}_{1} e^{i(m \hat{z}-\sigma \hat{t})},
$$

$$
\hat{q}(\hat{z}, \hat{t})=1+\hat{q}_{1} e^{i(m \hat{z}-\sigma \hat{t})},
$$

where $m$ is the (real) dimensionless wavenumber and $\sigma$ is the (complex) dimensionless growth rate, Sisoev et al. obtain a dispersion relation for $\sigma$ satisfying

$$
\sigma^{2}+\left(i a_{1,0}-a_{1,1} m\right) \sigma+\frac{m}{1+\varepsilon}\left(-a_{0,3} m^{3}-a_{0,1} m+i a_{0,0}\right)=0
$$

where $\varepsilon=h_{0} / r_{f}$ and the constant coefficients $a_{1,0}, a_{1,1}, a_{0,3}, a_{0,1}, a_{0,0}$ are defined in the Appendix. The variables $\hat{h}, \hat{z}$, and $\hat{t}$ are dimensionless quantities satisfying the scalings

$$
h=h_{0} \hat{h}, \quad z=\frac{h_{0}}{\kappa} \hat{z}, \quad t=\frac{h_{0}}{\kappa U} \hat{t},
$$

where

$$
\kappa=\left(\frac{\rho g h_{0}^{2}}{\gamma}\right)^{1 / 3}, \quad U=\frac{Q}{2 \pi r_{f} h_{0}},
$$

represent a stretching parameter and a characteristic velocity scale, respectively. We note that the long-wave model used by Sisoev et al. is derived under the assumption that $\kappa^{2} \ll 1$, and that the model's accuracy decreases with increasing values of $\kappa^{2} \ll 1$ [30]. Another important parameter,

$$
\delta=\frac{1}{45 \nu^{2}}\left(\frac{\rho g^{4} h_{0}^{11}}{\gamma}\right)^{1 / 3},
$$

is used to parametrize the falling film problem [18]. In order for the moderate-Reynolds-number model to apply, the waves must be sufficiently long, which for small values of $\delta$

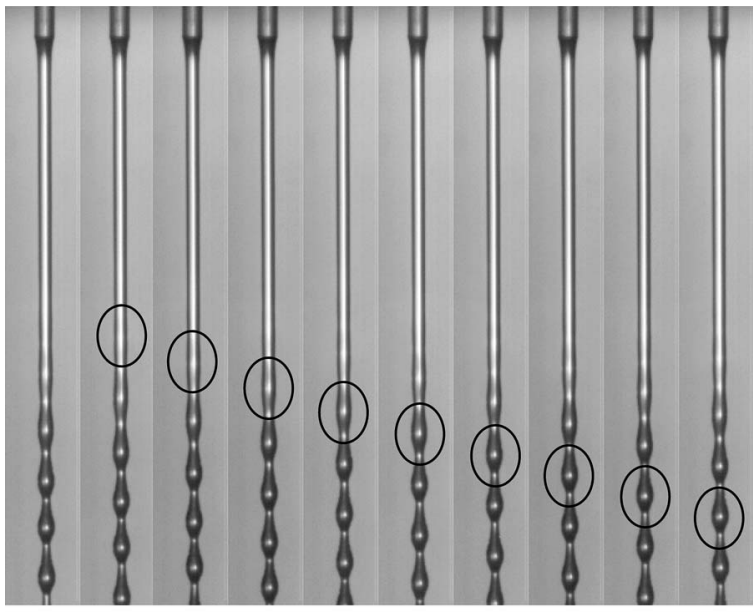
(a) (b)
(b) (c) (d)
(d) (e) (f)
(g) (h) (i) (j)

FIG. 4. An annular film of castor oil flowing down the outside of a thin nylon fiber $\left(r_{f}=0.029 \mathrm{~cm}\right)$. The film loses uniformity approximately $5.4 \mathrm{~cm}$ from the orifice indicated by the circled area in frame (b). Subsequent frames track the position and growth of this nascent perturbation. Time between images is $0.42 \mathrm{~s}$ and elapsed time $=3.7 \mathrm{~s}$. Image height $=9.7 \mathrm{~cm}$. 

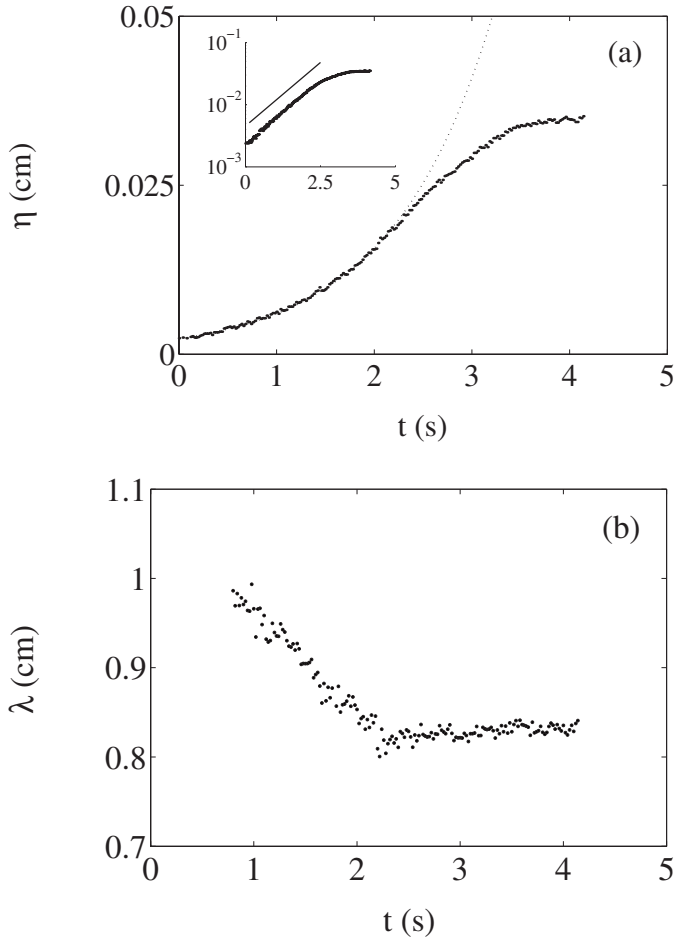

FIG. 5. (a) Amplitude ( $\eta$ ) and (b) wavelength $(\lambda)$ of a nascent perturbation as a function of time for castor oil $\left(Q=0.0194 \mathrm{~cm}^{3} / \mathrm{s}\right)$; experimental data correspond to the perturbation tracked in Fig. 4. (a) The initial amplitude growth of the perturbation is exponential (shown in inset on log-linear scale) followed by nonlinear saturation. Dotted line: fit of data to $\eta(t)$ $=0.0023 e^{0.959 t}$ corresponding to growth between frames $(\mathrm{b})$ and $(\mathrm{g})$ in Fig. 4. (b) The wavelength decreases during the time interval that the amplitude grows exponentially and then saturates in length as the amplitude saturates in size.

requires $\varepsilon \leq 1$. The values of $\delta, \varepsilon$, and $\kappa^{2}$ for our experiments are provided in Table III.

The stability results developed by Craster and Matar [15] and Sisoev et al. [18] are from a temporal analysis (since $m \in \mathbb{R}$ and $\sigma \in \mathrm{C}$ ), and thus model the case in which interfacial perturbations grow in amplitude everywhere along the film [31]. Our interest is in testing these stability predictions by comparing the theoretical dispersion relations (8) and (10) to the growth rate of perturbations measured in experiments conducted in the Stokes and moderate-Reynolds-number flow limits.

TABLE III. Experimental values of parameters from the moderate-Reynolds-number model [18].

\begin{tabular}{cccc}
\hline \hline Fluid & $\delta$ & $\varepsilon$ & $\kappa^{2}$ \\
\hline Vegetable oil $^{\mathrm{a}}$ & $0.013-0.028$ & $2.6-3.3$ & $0.28-0.38$ \\
Glycerol solution $^{\mathrm{a}}$ & $0.035-0.054$ & $2.9-3.3$ & $0.27-0.32$ \\
Glycerol solution $^{\mathrm{b}}$ & $0.033-0.043$ & $8.2-8.8$ & $0.26-0.29$ \\
\hline \hline
\end{tabular}

${ }^{\mathrm{a}} r_{f}=0.029 \mathrm{~cm}$.

${ }^{\mathrm{b}} r_{f}=0.010 \mathrm{~cm}$.
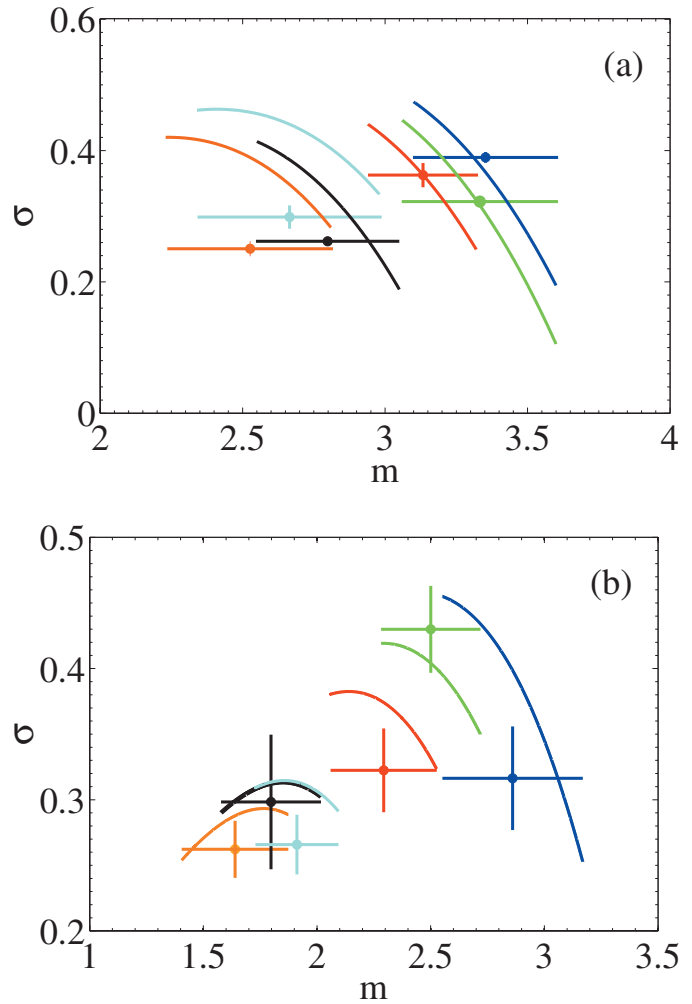

FIG. 6. (Color) Dimensionless growth rate as a function of dimensionless wavenumber for experiments with (a) castor oil and (b) vegetable oil. Circles correspond to experimental data for the average growth rate and wavenumber measured over several perturbations at (a) $\mathrm{Q}=0.0194$, orange; 0.0171, black; 0.0148, cyan; 0.0123, red; 0.0111, green; and 0.0103, blue; (b) $\mathrm{Q}=0.416$, orange; 0.383, black; 0.342 , cyan; 0.252 , red; 0.211 , green; and 0.170 , blue. Units are $\mathrm{cm}^{3} / \mathrm{s}$. Vertical bars represent the standard deviation of $\sigma$ and horizontal bars represent the range of $m$ measured during the period of exponential growth over all perturbations measured. Corresponding colored curves represent the real part $\operatorname{Re}(\sigma)$ predicted by the Stokes model (8) plotted over the range of $m$ measured in experiments [15].

\section{B. Experimental observations of perturbation formation}

Figure 4 shows a series of images tracking the formation of a perturbation along an annular film of castor oil. In Fig. 4(b), a small-amplitude perturbation first appears along the film approximately $5.4 \mathrm{~cm}$ from the orifice (circled region). Figures $4(\mathrm{c})-4(\mathrm{j})$ track the position of this perturbation as it grows in amplitude and saturates in size. Once formed, the perturbation continues moving down the fiber (not shown). Since the perturbation grows in amplitude as it travels down the fiber, the flow is spatially unstable rather than temporally unstable to perturbations [31].

To characterize the growth of a perturbation, we measure the amplitude $\eta$ (half the radial distance from first minimum to first maximum) and the wavelength $\lambda$ (the axial distance from first to second maximum) as shown in Fig. 2(b) using edge-detection software; both measurements are made in the moving reference frame of the perturbation. The data shown in Fig. 5 correspond to the perturbation tracked in Fig. 4. Figure 5(a) shows that the nascent growth of the amplitude is 

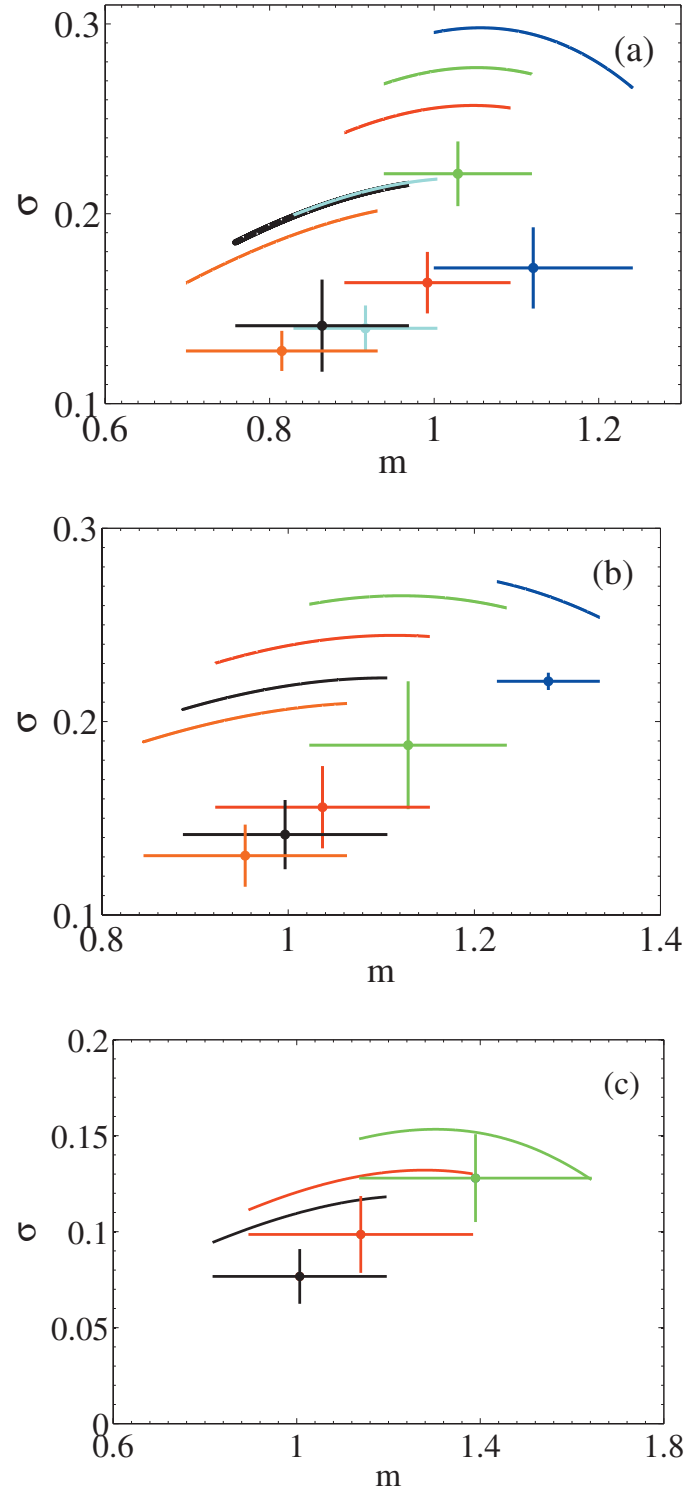

FIG. 7. (Color) Dimensionless growth rate as a function of dimensionless wavenumber for experiments with (a) vegetable oil, (b) glycerol solution with $r_{f}=0.029 \mathrm{~cm}$, and (c) glycerol solution with $r_{f}=0.010 \mathrm{~cm}$. Circles correspond to experimental data of the average growth rate and wavenumber measured over several perturbations at (a) $\mathrm{Q}=0.416$, orange; 0.383, black; 0.342, cyan; 0.252, red; 0.211 , green; and 0.170 , blue; (b) $\mathrm{Q}=0.538$, orange; 0.493 , black; 0.437 , red; 0.381, green; and 0.325 , blue; (c) $\mathrm{Q}=0.548$, black; 0.444 , red; 0.341 , green. Units are $\mathrm{cm}^{3} / \mathrm{s}$. Vertical bars represent the standard deviation of $\sigma$ and horizontal bars represent the range of $m$ measured during the period of exponential growth over all perturbations measured. Corresponding colored curves represent the imaginary part $\operatorname{Im}(\sigma)$ predicted by the moderate-Reynolds-number model (10) plotted over the range of $m$ measured in experiments [18].

exponential (inset), followed by a slower phase as the perturbation saturates in size $\left(\eta_{\text {saturate }}=0.035 \mathrm{~cm}\right)$. The growth rate for the initial formation of the amplitude is determined from a least-squares fit of the data to an exponential function, yielding the dimensional growth rate $\sigma_{\text {dim }}=0.959 \mathrm{~s}^{-1}$ [fit indicated by dotted line in Fig. 5(a)]. The wavelength of this

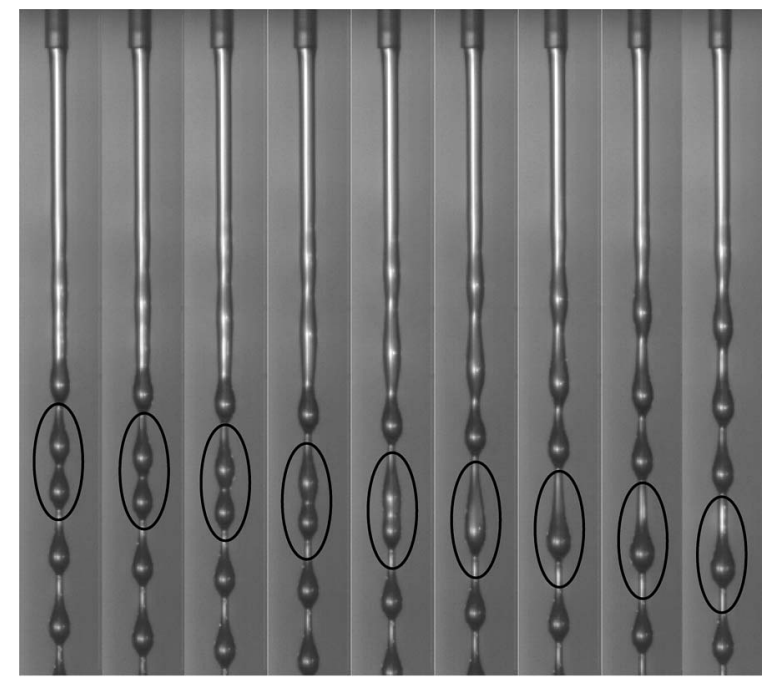

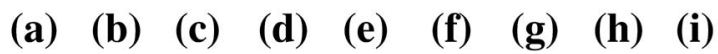

FIG. 8. Coalescence of two perturbations along an annular film of glycerol solution. $Q=0.359 \mathrm{~cm}^{3} / \mathrm{s}, r_{f}=0.029 \mathrm{~cm}$, time between images is $0.0125 \mathrm{~s}$, elapsed time $=0.1 \mathrm{~s}$, image height $=9.7 \mathrm{~cm}$.

perturbation decreases from $\lambda=0.98 \mathrm{~cm}$ to $0.80 \mathrm{~cm}$ during the time interval in which the amplitude grows exponentially $(0 \leq t \leq 2.34 \mathrm{~s})$, before saturating in length to $\lambda=0.83 \mathrm{~cm}$, as shown in Fig. 5(b). The variation in $\lambda$ during the exponential phase of growth indicates that the annular film is unstable to a range of wavenumbers $(=2 \pi / \lambda)$, rather than to one fixed value.

The initial exponential growth of the amplitude displayed in Fig. 5 is typical of observations made in the castor oil, vegetable oil, and glycerol solution experiments. Next, we compare the measured growth rates to predictions developed from linear stability theory. Comparison of perturbation growth to the Stokes (8) or moderate-Reynolds-number (10) dispersion relations depend on the flow conditions in each experiment, specifically on the Reynolds and Bond numbers (provided in Table II). In the castor oil experiments, Bo $\sim O\left(10^{-1}\right)$ and $\operatorname{Re} \sim O\left(10^{-2}\right)$, thus satisfying the requirements of the Stokes model $[\mathrm{Bo} \ll 1, \operatorname{Re} \leq O(1)]$. Since 25 $\lesssim \operatorname{Re} \lesssim 30$ in the glycerol solution experiments, inertial effects cannot be ignored, and so we compare this case to the moderate-Reynolds-number model. With Bo $O\left(10^{-1}\right)$ and $9.4<\operatorname{Re}<11.6$, the vegetable oil experiments are on the border of the requirements for the Stokes model. In this case we compare the experimental data to the Stokes and moderateReynolds-number dispersion relations.

Figure 6 shows a comparison of the measured amplitude growth rate to the dispersion relation developed by Craster and Matar in the Stokes flow limit (8) for (a) castor oil and (b) vegetable oil at various flow rates. At a given flow rate, the growth rate for several perturbations was measured (8-12 perturbations for castor oil, and 15-44 for vegetable oil). The average dimensionless growth rate $\left(\sigma=\sigma_{\text {dim }} \mathcal{L} / \mathcal{V}\right)$ and dimensionless wavenumber $(m=2 \pi \mathcal{L} / \lambda)$ measured over all the perturbations are denoted by a circle, with each color corresponding to a different flow rate. The vertical bars represent the standard deviation of all the growth rates measured at a 


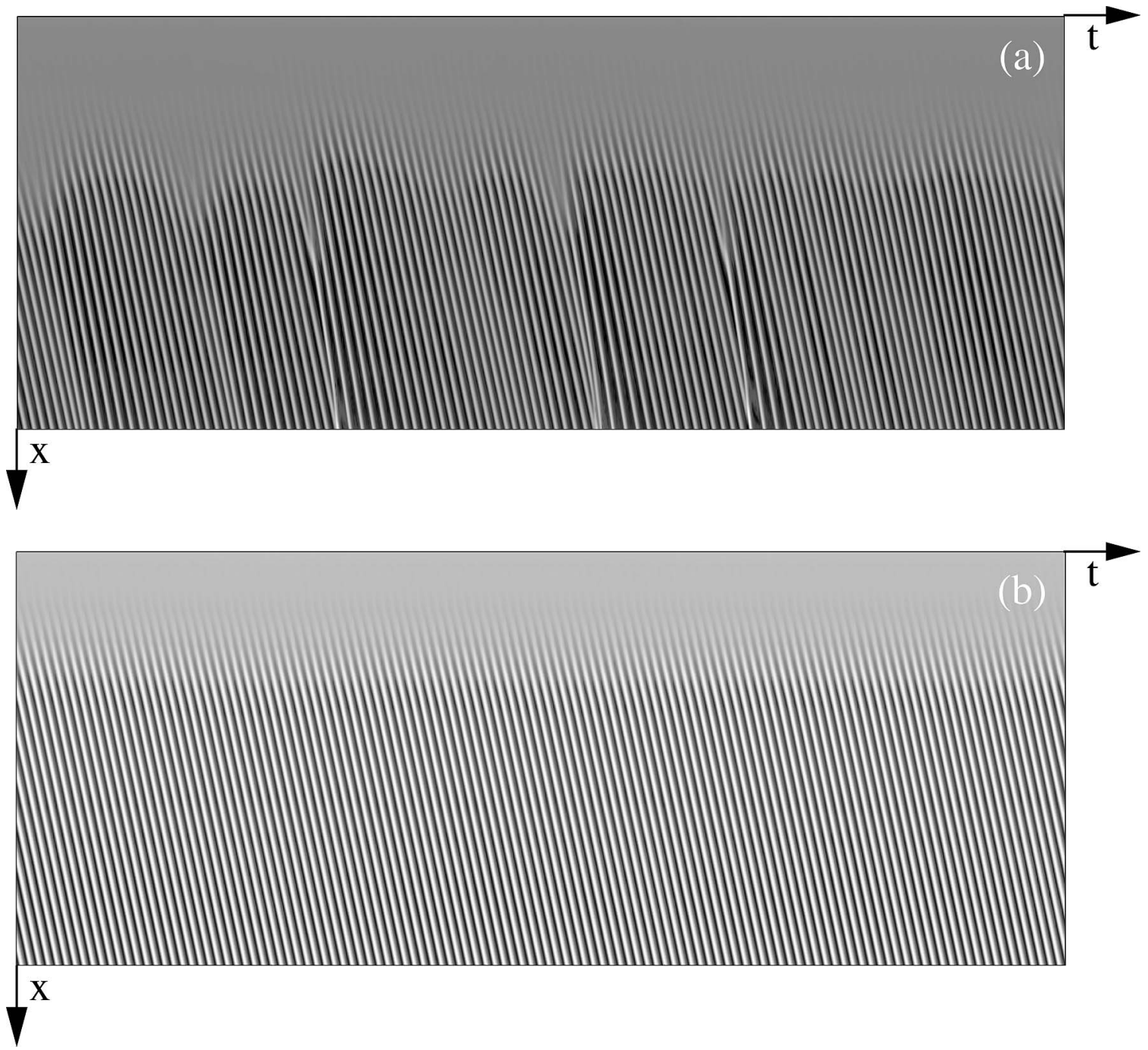

FIG. 9. Space-time plot illustrating the motion of perturbations along a vertical fiber $\left(r_{f}=0.029 \mathrm{~cm}\right)$ during (a) unsteady and (b) steady behavior. Lighter gray level corresponds to thicker regions of the fluid interface and darker gray level corresponds to thinner regions; the light characteristic lines indicate the location of perturbations along the fiber as a function of time. Experimental fluid: glycerol solution $\left(Q_{c}=0.345 \mathrm{~cm}^{3} / \mathrm{s}\right)$, (a) $Q=0.347 \mathrm{~cm}^{3} / \mathrm{s}$, (b) $Q=0.336 \mathrm{~cm}^{3} / \mathrm{s}$, elapsed time $=8.09 \mathrm{~s}$, image height $=8.22 \mathrm{~cm}$. The top of each image is 0.58 $\mathrm{cm}$ below the orifice.

given flow rate. Since the perturbation wavelength varies during the exponential phase of growth, we cannot assign a single wavenumber to its growth. Instead, the horizontal bars represent the range of wavenumber measured during the period of exponential growth of all the perturbations. The corresponding colored curves represent the real part of the growth rate predicted by (8) plotted over the range of wavenumber measured at each flow rate. We consider the theory to be in agreement with the experimental data (at a given flow rate) if the theoretical curve overlaps the rectangular region defined by the resolution bars of the data. Figure 6 shows that the Stokes theory is in agreement with four of the six castor oil experiments and with five of the six vegetable oil experiments. In the other three experiments, the theory overestimates the measured values by 1-8\%. Overall, the quantitative agreement between theory and experimental data is excellent, a significant result considering that (i) the comparison is between a temporal stability theory and a spa- tial instability of the film, and (ii) the value of the Reynolds number in the vegetable oil experiments, $\operatorname{Re} \approx 10$, is slightly higher than the criterion for the Stokes model, $\operatorname{Re} \leq O(1)$.

Figure 7 shows a comparison of the measured amplitude growth rate to the dispersion relation developed by Sisoev et $a l$. in the moderate-Reynolds-number limit (10) for (a) vegetable oil, (b) glycerol solution with $r_{f}=0.029 \mathrm{~cm}$, and (c) glycerol solution with $r_{f}=0.010 \mathrm{~cm}$ at various flow rates. The data and theory are presented in a similar fashion to Fig. 6 with the exception that the dimensionless growth rate and wavenumber are given by $\sigma=\sigma_{\mathrm{dim}} h_{0} /(\kappa U)$ and $m$ $=2 \pi h_{0} /(\kappa \lambda)$, and the growth rates for the glycerol solution experiments are averaged over 88-102 perturbations for $r_{f}$ $=0.029 \mathrm{~cm}$ and $17-40$ perturbations for $r_{f}=0.010 \mathrm{~cm}$. In the case of the thicker fiber $\left(r_{f}=0.029 \mathrm{~cm}\right)$, the moderateReynolds-number model overestimates the measured growth rates by $12-38 \%$ for vegetable oil and $13-30 \%$ for glycerol solution, as shown in Figs. 7(a) and 7(b). In the case of the 


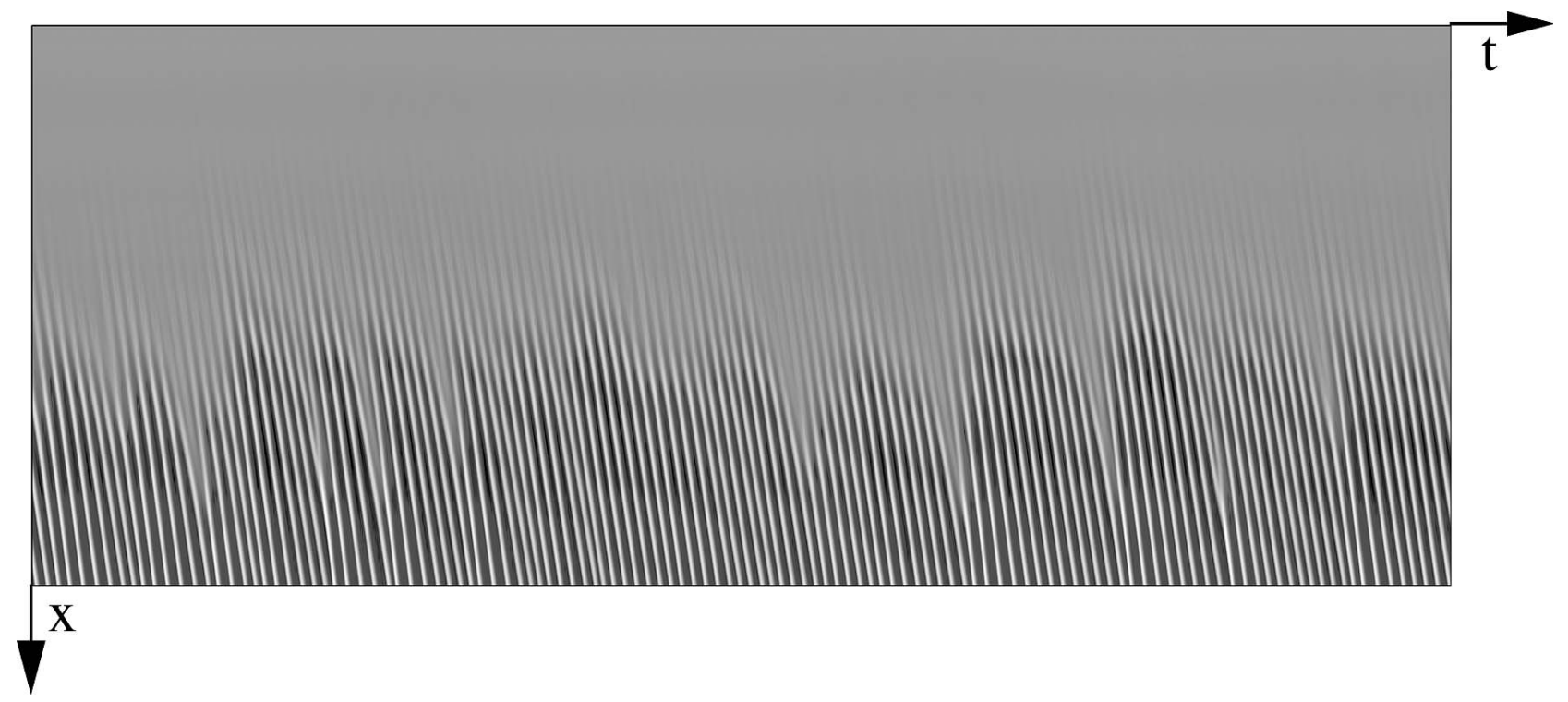

FIG. 10. Space-time plot illustrating the motion of perturbations along a vertical fiber $\left(r_{f}=0.029 \mathrm{~cm}\right)$ during unsteady behavior. Experimental fluid: glycerol solution; $Q=0.516 \mathrm{~cm}^{3} / \mathrm{s}$, elapsed time $=8.09 \mathrm{~s}$, image height $=8.22 \mathrm{~cm}$. The top of the image is $0.58 \mathrm{~cm}$ below the orifice.

thinner fiber $\left(r_{f}=0.010 \mathrm{~cm}\right)$, the model agrees with two of the glycerol solution experiments and overestimates the third experiment by $4 \%$ [see Fig. 7(c)]. We make a few observations based on the data shown in Figs. 6 and 7 .

(1) Comparing Figs. 6(b) and 7(a), we find that the Stokes model more accurately predicts the growth rate of the vegetable oil perturbations than the moderate-Reynolds-number model (note that the experimental data are the same in these two figures).

(2) The fiber in the glycerol solution experiments shown in Fig. 7(b) was slightly off center, which may explain the discrepancy between theory and data; however, the percentage error is larger in the vegetable oil experiments [Fig. 7(a)] where the fiber was on center.

(3) Recall that the long-wave moderate-Reynolds-number model applies if (i) $\kappa^{2} \ll 1$ and (ii) $\varepsilon \leq 1$ for small values of $\delta$. In the experiments shown in Fig. $7, \kappa^{2} \sim O\left(10^{-1}\right), \varepsilon>1$, and $\delta \sim O\left(10^{-2}\right)$ (see Table III). We conclude that the parameter range of the experiments is out of the range for which the moderate-Reynolds-number model applies, thus explaining the discrepancy between theory and data in Fig. 7.

(4) The range of the measured amplitude growth rate in experiments (indicated by the vertical bars in Figs. 6 and 7) varies by fluid and flow rate. For castor oil [Fig. 6(a)], the range is fairly small which we attribute to the low Reynolds number $\left[\operatorname{Re} \sim O\left(10^{-2}\right)\right]$ in the experiments. In 13 of the 14 data sets shown in Fig. 7, the range of growth rates is large. Naively, one could attribute this to the higher Reynolds number in the experiments $(10 \lesssim \operatorname{Re} \lesssim 30)$. This, however, is not the complete picture. Notice that the range is significantly smaller for the glycerol solution experiment at $Q$ $=0.325 \mathrm{~cm}^{3} / \mathrm{s}$ [blue data set in Fig. 7(b)]. The difference in this experiment compared to the other glycerol solution and vegetable oil experiments is in the behavior of the perturbations. The perturbation behavior in the experiments with a large range of growth rates is unsteady, while the behavior in the glycerol solution experiment shown in blue is steady. (The notion of unsteady and steady perturbation behavior will be explained in detail in Sec. IV.) Therefore, we find the range of measured growth rates to be correlated with the Reynolds number of the flow and the longer-time dynamics of the perturbations.

Next, we examine the dynamics of perturbations after their initial formation and explain the physical mechanism that controls the known transition in the flow from unsteady to steady perturbation behavior.

\section{STEADY AND UNSTEADY PERTURBATION DYNAMICS}

The dynamics of interfacial perturbations along an annular film flowing down a vertical fiber can be broken down into three essential stages: (i) initial exponential growth of the perturbation amplitude accompanied by variation in the wavelength; (ii) nonlinear saturation of the perturbation amplitude and wavelength; and (iii) longer-time behavior in which the perturbation wavelength may (unsteady-see Fig. 1) or may not (steady) vary along the fiber; this last stage has been noted in other experimental studies [14,15,19]. Here we explain the physical mechanism that controls this third stage of dynamics.

In experiments with the thicker fiber $\left(r_{f}=0.029 \mathrm{~cm}\right)$, we observe that the perturbation motion abruptly transitions from unsteady (regime $a$ ) to steady (regime $b$ ) behavior at a critical flow rate $Q_{c}$ for all three fluids $\left(Q_{c}=0.0095 \mathrm{~cm}^{3} / \mathrm{s}\right.$ for castor oil, $0.119 \mathrm{~cm}^{3} / \mathrm{s}$ for vegetable oil, and $0.345 \mathrm{~cm}^{3} / \mathrm{s}$ for glycerol solution) [26], similar to observations made by Duprat et al. in their experiments with silicone oil [19]. Following KDB [14], we define the flow to be steady if no perturbations coalesce as they travel down the full length of the fiber $(\approx 2 \mathrm{~m})$, and unsteady otherwise, while the flow is jetting from the orifice. An example of 
unsteady behavior in which two perturbations coalesce is shown in Fig. 8. Note that we will not be examining the dripping state, regime $c$ [14], which occurs at a lower flow rate $Q_{\text {drip }}<Q_{c}$. We find that the transition from unsteady $\left(Q>Q_{c}\right)$ to steady $\left(Q_{\text {drip }}<Q<Q_{c}\right)$ behavior is robust in the sense that, once an experiment transitions to steady behavior (as the flow rate decreases), it does not revert back to the unsteady state. We note that steady perturbation behavior was not observed in the glycerol solution experiments with the thinner fiber $\left(r_{f}=0.010 \mathrm{~cm}\right)$; instead, the flow transitioned directly from regime $a$ to $c$. Since our focus is on the transition from unsteady to steady dynamics, we will not consider this case further.

The space-time plots in Fig. 9 illustrate (a) unsteady and (b) steady perturbation behavior for experiments with glycerol solution $\left(r_{f}=0.029 \mathrm{~cm}\right)$. Each plot is focused on 8.22 $\mathrm{cm}$ of the film, with the top of each plot located $0.58 \mathrm{~cm}$ below the orifice; the time span of each plot is $8.09 \mathrm{~s}$. The plots are created by mapping the radius of the free surface of the film, $r=S(z, t)$, to a gray level, with lighter gray corresponding to thicker regions of the free surface and darker gray corresponding to thinner regions. The light characteristic lines in the plots indicate the location of perturbations as they move down the fiber, and their slopes represent the speed of the perturbations. Two features in the space-time plots distinguish the unsteady and steady perturbation behavior. First, the location along the fiber where perturbations form (which we refer to as the boundary) oscillates irregularly in the unsteady case and appears nearly fixed in the steady case [26]. Second, in the unsteady case, perturbations coalesce as faster-moving perturbations collide into slowermoving perturbations (indicated by intersecting characteristic lines), whereas in the steady case perturbations do not coalesce as they travel with the same terminal speed down the fiber (indicated by parallel characteristic lines) $[14,26]$. The longer-time motion of the perturbations appears to be correlated with the motion of the boundary. Notice in Fig. 9(a) that large spatial variations in the boundary modulate the perturbation speed (i.e., the slope of the characteristic lines), which results in coalescence events later down the fiber. In the steady case, there is no spatial variation in the boundary, and, as a result, the perturbations remain equally spaced as they travel with constant terminal speed down the full length of the fiber [not shown in Fig. 9(b)]. Our observations of the steady case (regime $b$ ) are consistent with those of KDB [14]. Given the robustness of the steady dynamics in all of our experiments with the thicker fiber, we conclude that this is not a transient state as CM report [15]. Finally, we note that, when the flow is unsteady, the oscillation frequency of the boundary increases with increasing flow rate; for example, compare the boundary frequencies in Fig. 9(a) $\left(Q=0.347 \mathrm{~cm}^{3} / \mathrm{s}\right)$ and Fig. $10\left(Q=0.516 \mathrm{~cm}^{3} / \mathrm{s}\right)$.

To characterize the motion of the boundary, we measure the distance from the orifice $D$ at which each perturbation initially forms along the fiber (at a fixed flow rate) using edge-detection software; a perturbation is detected when its amplitude $(\eta)$ initially exceeds $1 / 10$ of a pixel or $\approx 0.002 \mathrm{~cm}$. The data shown in Fig. 11(a) correspond to the space-time plots of the unsteady $(\bullet)$ and steady $(+)$ experi-
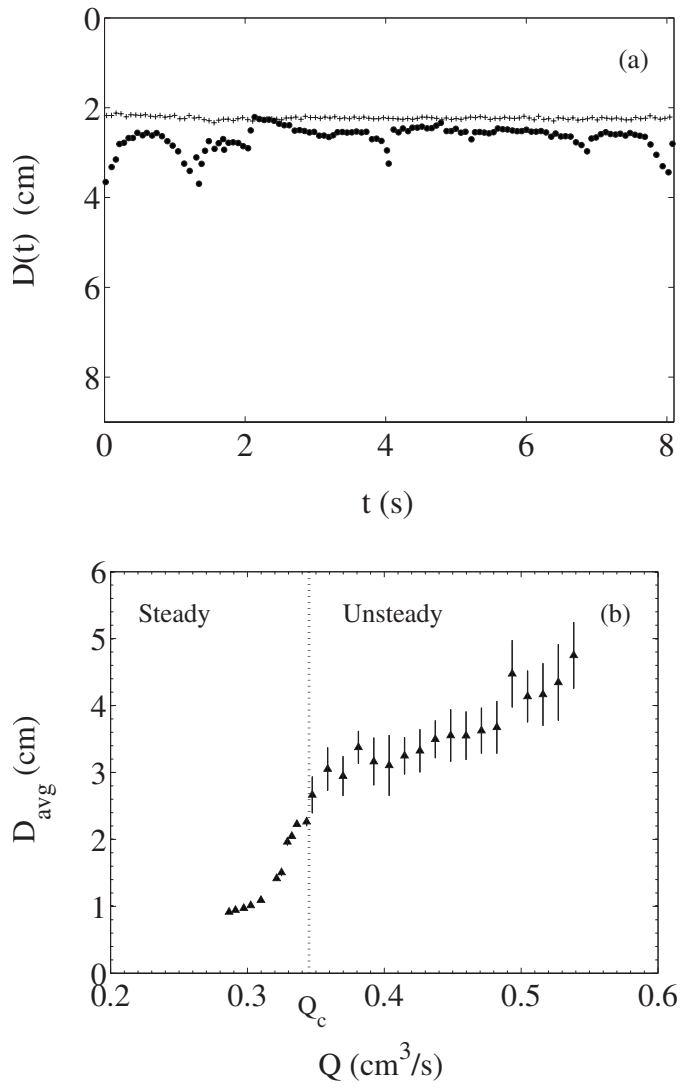

FIG. 11. (a) The distance from the orifice $(D)$ where perturbations initially form along the fiber (i.e., the location of the boundary) as a function of time, corresponding to the data shown in Fig. 9(a) for the unsteady case at $Q=0.347 \mathrm{~cm}^{3} / \mathrm{s}$ (O) and in Fig. 9(b) for the steady case at $Q=0.336 \mathrm{~cm}^{3} / \mathrm{s}(+)$. (b) Average distance $\left(D_{\text {avg }}\right)$ from the orifice at which perturbations form as a function of flow rate $Q$ for experiments with glycerol solution $\left(r_{f}=0.029 \mathrm{~cm}\right)$. Vertical bars represent the standard deviation of $D$ over all the perturbations measured at a given flow rate. The dotted vertical line denotes the transition flow rate separating steady and unsteady perturbation behavior.

ments shown in Fig. 9. Figure 11(b) is a plot of the average distance from the orifice at which perturbations form, $\left(D_{\mathrm{avg}}\right)$, as a function of flow rate in experiments with glycerol solution. The vertical bars represent the standard deviation of $D$ over all the perturbations measured at a fixed flow rate, and the dotted vertical line represents the transition flow rate $Q_{c}$. The distance from the orifice at which perturbations form increases monotonically with increasing flow rate. In the steady case, at a given flow rate the distance is nearly constant, whereas in the unsteady case, the range of distance over which perturbations form increases with increasing flow rate. These results are consistent with experimental observations made by Duprat et al. [19].

To understand the physical mechanism controlling the steady and unsteady states, we examine the power spectra of $D(t)$ in the glycerol solution experiments. Figures 12(a) and 12(b) represent the power spectra for the steady (+) and unsteady (@) perturbation behavior shown in Fig. 11(a). In the steady case the fundamental frequency $\left(f_{0}\right)$, which is the first harmonic of the power spectrum, represents the rate at which 

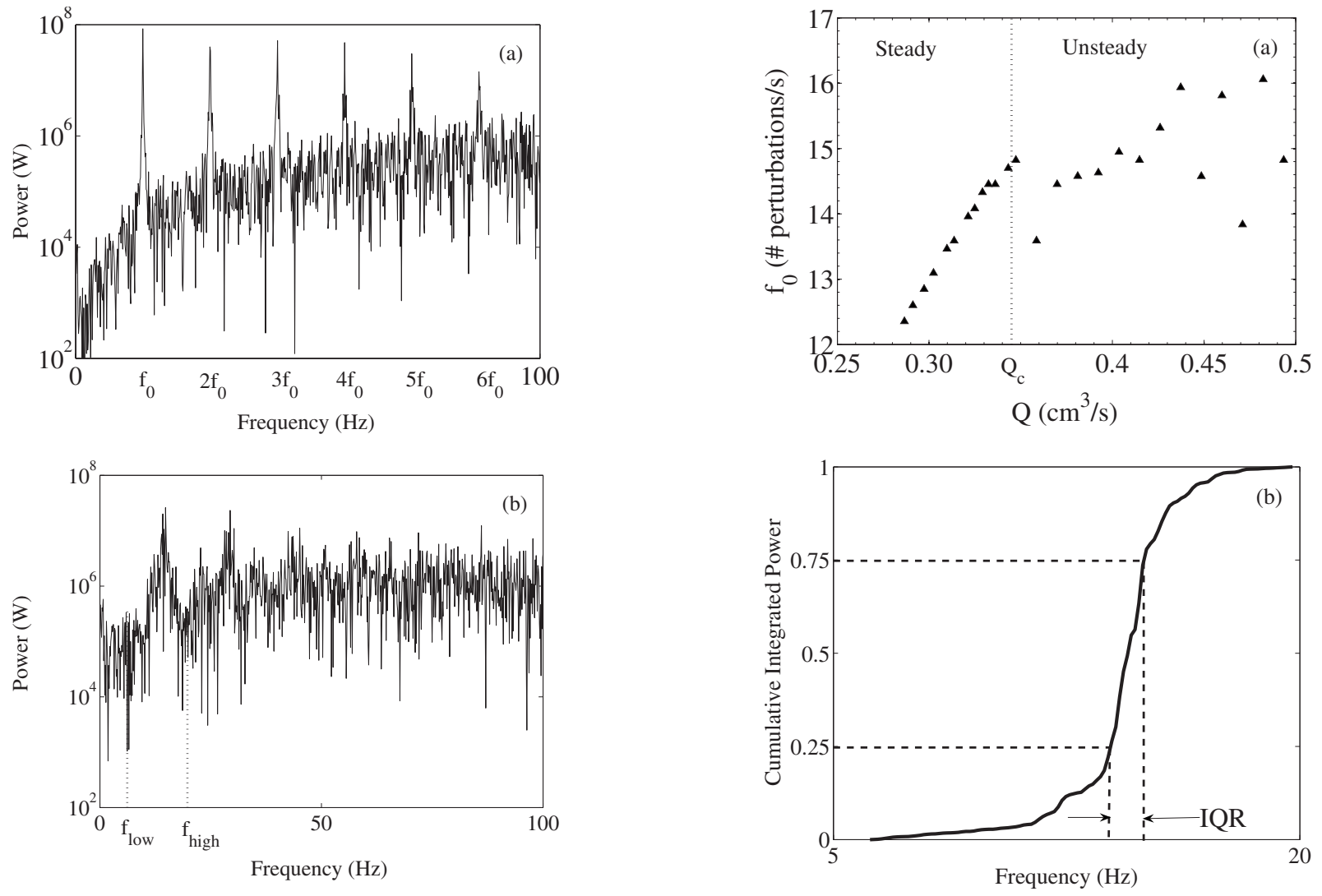

FIG. 12. Power spectrum of the distance from the orifice $D$ at which perturbations form as a function of time. Plots correspond to the data shown in Fig. 11(a) for (a) steady behavior (+) at $Q=0.336 \mathrm{~cm}^{3} / \mathrm{s}$ and (b) unsteady behavior (O) at $Q=0.347 \mathrm{~cm}^{3} / \mathrm{s}$ for glycerol solution. In the steady case, the fundamental frequency $f_{0}=14.45 \mathrm{~Hz}$ is the first harmonic in the power spectrum. In the unsteady case, the bandwidth supporting the fundamental peak is larger than in the steady case.

perturbations form along the fiber [e.g., $f_{0}=14.45$ perturbations/s in the experiment shown in Fig. 9(b)]; in the unsteady case the fundamental peak is much broader so that $f_{0}$ is less well defined. As a function of increasing flow rate, the fundamental frequency (i.e., rate of perturbation formation) increases linearly when the perturbation dynamics are steady $\left(Q<Q_{c}\right)$, and is scattered over about $\approx 15$ perturbations/s when the dynamics are unsteady $\left(Q>Q_{c}\right)$ [see Fig. 13(a)]. Another feature in the power spectra distinguishing steady and unsteady behavior is in the frequency bandwidth supporting the fundamental peak; the bandwidth of the unsteady spectrum is larger than the steady spectrum in Fig. 12. We characterize the bandwidth of the fundamental peak by measuring the interquartile region (IQR). The IQR is defined as the frequency bandwidth bounding the middle $50 \%$ of the (normalized) cumulative integrated power under the fundamental peak; an example is shown in Fig. 13(b) corresponding to the power spectra in Fig. 12(b) [32]. The $\mathrm{IQR}$, or bandwidth, measures the modulation of the fundamental frequency, or more physically, the modulation of the rate at which perturbations form along the fiber. A jump in the bandwidth occurs at the transition flow rate $Q_{c}$ in the glycerol solution experiments, as shown in Fig. 14. For

FIG. 13. (a) Fundamental frequency as a function of flow rate measured from power spectra of $D$ versus $t$ data in experiments with glycerol solution $\left(r_{f}=0.029 \mathrm{~cm}\right)$. The dotted vertical line denotes the transition flow rate separating steady and unsteady perturbation behavior. (b) The (normalized) cumulative integrated power measured within the support of the first peak between $f_{\text {low }}$ $=6.18 \mathrm{~Hz}$ and $\mathrm{f}_{\text {high }}=19.77 \mathrm{~Hz}$ for the spectra shown in Fig. 12(b). The interquartile region is the frequency bandwidth bounding the middle $50 \%$ of the cumulative integrated power.

$Q<Q_{c}$, the bandwidth is nearly zero, thus the rate of perturbation formation is nearly constant, resulting in longer-time steady perturbation behavior. For $Q>Q_{c}$, the bandwidth is sizable and increases with increasing flow rate; thus there is a significant modulation of the rate at which perturbations form. It is this large modulation that results in the longertime unsteady dynamics of the perturbations. While the transition in Fig. 14 is striking, it is not entirely clear whether it is a subcritical or supercritical transition, and, if subcritical, whether the transition is hysteretic.

\section{CONCLUSIONS}

In an experimental study, we examine the motion of an annular viscous film flowing under the influence of gravity down the outside of a vertical fiber. We find the unperturbed flow in our experiments is well approximated by a steady, unidirectional, parallel flow. The dynamics of the perturbed flow can be divided into three stages: (i) initial exponential growth of the perturbation amplitude accompanied by variation in the perturbation wavelength; (ii) nonlinear saturation 


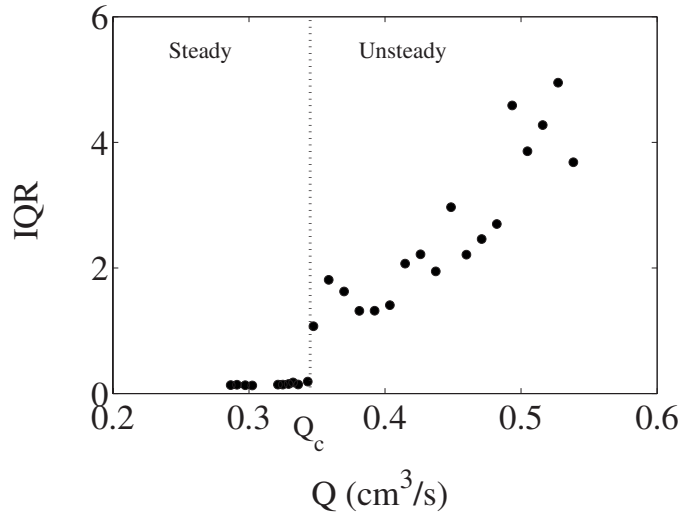

FIG. 14. Interquartile region, or frequency bandwidth, as a function of flow rate for the glycerol solution experiments $\left(r_{f}\right.$ $=0.029 \mathrm{~cm}$ ). An abrupt transition in the bandwidth occurs at the transition flow rate $Q_{c}$.

of the perturbation amplitude and wavelength; and (iii) longer-time behavior in which the perturbation wavelength may (unsteady) or may not (steady) vary along the film. During the first stage, we find that linear stability theory results developed from a long-wave Stokes flow model [15] are in excellent agreement with the initial growth of perturbations measured in experiments. The agreement between linear stability results developed from a moderate-Reynolds-number model [18] and experimental data depends on the fiber size; in particular, the agreement is excellent for a thin fiber and fair for a thicker fiber. The parameter range of our experiments likely exceeds the requirements of the moderateReynolds-number model, which explains these mixed results and highlights the need for more analysis of annular flows in the moderate-Reynolds-number limit.

A close examination of the longer-time steady and unsteady behavior of interfacial perturbations is shown to be correlated with (i) the rate of exponential growth of the perturbation amplitude; and (ii) the location along the fiber where perturbations initially form. In particular, we find that the rate of growth of the amplitude and the location along the fiber where perturbations form are nearly constant for the steady case, and vary over a range of values in the unsteady case. Furthermore, we find that the transition in the longertime perturbation dynamics from unsteady to steady behavior at a critical flow rate is correlated with a transition in the rate at which perturbations naturally form along the free surface of the film. In the steady case, the rate of perturbation formation is nearly constant, resulting in the perturbations remaining equally spaced as they travel with the same terminal speed down the fiber. In the unsteady case, the rate of perturbation formation is modulated, which results in the modulation of the initial speed and spacing between perturbations and ultimately leads to the coalescence of perturbations further down the fiber. It is not clear whether this transition is subcritical or supercritical, and, if subcritical, whether the transition is hysteretic.

We would like to thank A. Belmonte, M. G. Forest, M. Frey, D. Henderson, H. Segur, H. Stone, and T. Witelski for many helpful discussions and Timothy Baker for his aid in building the experimental apparatus. The authors acknowledge support from NSF REU Grants No. PHY-0097424 and No. PHY-0552790. L.B.S. also acknowledges support from NSF Grant No. DMS-0707755.

\section{APPENDIX}

The formulas for the constant coefficients described in the moderate-Reynolds-number dispersion relation (10) derived by Sisoev et al. [18] are

$$
\begin{aligned}
a_{0,0}= & -\frac{1}{5 \delta_{\varepsilon}}\left(1+\frac{\varepsilon}{2}\right)\left(3+\phi_{1}\right), \quad a_{0,3}=\frac{1}{5 \delta_{\varepsilon}}\left(1+\frac{\varepsilon}{2}\right), \\
a_{0,1}= & \frac{b_{0}}{16 \varepsilon^{5}[\phi(\varepsilon)]^{2}}\left(\frac{b_{1}}{b_{0}}-2 \phi_{1}-6\right)-\frac{1}{5 \delta_{\varepsilon}}\left(1+\frac{\varepsilon}{2}\right) \\
& \times\left(\frac{\varepsilon}{\kappa(1+\varepsilon)}\right)^{2}, \\
a_{1,1} & =\frac{2 b_{0}}{16 \varepsilon^{5}[\phi(\varepsilon)]^{2}}, \quad a_{1,0}=\frac{1}{5 \delta_{\varepsilon}}\left(1+\frac{\varepsilon}{2}\right),
\end{aligned}
$$

where

$$
\begin{aligned}
b_{0}=2 & (1+\varepsilon)^{6}[\ln (1+\varepsilon)]^{2}+\left[2-3(1+\varepsilon)^{2}\right](1+\varepsilon)^{4} \ln (1+\varepsilon) \\
+ & \frac{17}{12}(1+\varepsilon)^{6}-\frac{5}{2}(1+\varepsilon)^{4}+\frac{5}{4}(1+\varepsilon)^{2}-\frac{1}{6}, \quad(\mathrm{~A} 1 \mathrm{~d}) \\
b_{1}= & \frac{17 \varepsilon}{2}(1+\varepsilon)^{5}-10 \varepsilon(1+\varepsilon)^{3}+\frac{5 \varepsilon}{2}(1+\varepsilon)+4 \varepsilon(1+\varepsilon)^{5} \\
& \times[\ln (1+\varepsilon)]^{2}\left(3+\frac{1}{\ln (1+\varepsilon)}\right)+\varepsilon\left[2-3(1+\varepsilon)^{2}\right](1 \\
& +\varepsilon)^{4} \ln (1+\varepsilon)\left(\frac{4}{1+\varepsilon}-\frac{6(1+\varepsilon)}{2-3(1+\varepsilon)^{2}}\right. \\
& \left.+\frac{1}{(1+\varepsilon) \ln (1+\varepsilon)}\right), \\
\phi_{1}= & \frac{\varepsilon(1+\varepsilon)^{3}[4 \ln (1+\varepsilon)+1]-\varepsilon-7 \varepsilon^{2}-9 \varepsilon^{3}-3 \varepsilon^{4}}{(1+\varepsilon)^{4} \ln (1+\varepsilon)-\varepsilon-\frac{7}{2} \varepsilon^{2}-3 \varepsilon^{3}-\frac{3}{4} \varepsilon^{4}}-3,
\end{aligned}
$$

$\phi(\varepsilon)=\frac{4(1+\varepsilon)^{4} \ln (1+\varepsilon)-\left\{\left[3(1+\varepsilon)^{2}-1\right]\left[(1+\varepsilon)^{2}-1\right]\right\}}{16 \varepsilon^{3}}$,

$$
\delta_{\varepsilon}=9 \delta[\phi(\varepsilon)]^{2}, \quad \delta=\frac{1}{45 \nu^{2}}\left(\frac{\rho g^{4} h_{0}^{11}}{\gamma}\right)^{1 / 3}, \quad \kappa=\left(\frac{\rho g h_{0}^{2}}{\gamma}\right)^{1 / 3} .
$$


[1] D. Quere, Annu. Rev. Fluid Mech. 31, 347 (1999).

[2] E. Ruckenstein, J. Colloid Interface Sci. 246, 393 (2002).

[3] A. de Ryck and D. Quere, J. Fluid Mech. 311, 219 (1996).

[4] A. de Ryck and D. Quere, Langmuir 14, 1911 (1998).

[5] A. Q. Shen, B. Gleason, G. H. McKinley, and H. A. Stone, Phys. Fluids 14, 4055 (2002).

[6] S. L. Goren, J. Fluid Mech. 12, 309 (1962).

[7] D. Quere, Europhys. Lett. 13, 721 (1990).

[8] H.-C. Chang and E. A. Demekhin, J. Fluid Mech. 380, 233 (1999).

[9] A. L. Frenkel, Europhys. Lett. 18, 583 (1992).

[10] S. Kalliadasis and H.-C. Chang, J. Fluid Mech. 261, 135 (1994).

[11] S. P. Lin and W. C. Liu, AIChE J. 21, 775 (1975).

[12] J. R. Lister, J. M. Rallison, A. A. King, L. J. Cummings, and O. E. Jensen, J. Fluid Mech. 552, 311 (2006).

[13] S. Zuccher, Exp. Fluids 39, 694 (2005).

[14] I. L. Kliakhandler, S. H. Davis, and S. G. Bankoff, J. Fluid Mech. 429, 381 (2001).

[15] R. V. Craster and O. K. Matar, J. Fluid Mech. 553, 85 (2006).

[16] F. J. Solorio and M. Sen, J. Fluid Mech. 183, 365 (1987).

[17] Y. Y. Trifonov, AIChE J. 38, 821 (1992).

[18] G. M. Sisoev, R. V. Craster, O. K. Matar, and S. V. Gerasimov, Chem. Eng. Sci. 61, 7279 (2006).

[19] C. Duprat, C. Ruyer-Quil, S. Kalliadasis, and F. Giorgiutti-
Dauphine, Phys. Rev. Lett. 98, 244502 (2007).

[20] J. Plateau, Statique Experimentale et Theorique des Liquides Soumis aux Seules Forces Moleculaires (Gauthier-Villars, Paris, 1873).

[21] W. S. Rayleigh, Proc. London Math. Soc. 10, 4 (1878).

[22] W. S. Rayleigh, Philos. Mag. 34, 145 (1892).

[23] C. Weber, Z. Angew. Math. Mech. 11, 136 (1931).

[24] S. Chandrasekhar, Hydrodynamic and Hydromagnetic Stability (Dover, New York, 1961).

[25] R. J. Donnelly and W. Glaberson, Proc. R. Soc. London, Ser. A 290, 547 (1966).

[26] L. Smolka, J. North, B. Guerra, Bull. Am. Phys. Soc. 51, GD.00006 (2006).

[27] J. Soria, W. K. Chiu, and M. P. Norton, Exp. Therm. Fluid Sci. 3, 291 (1990).

[28] W. Frei and C. C. Chen, IEEE Trans. Comput. 26, 988 (1977).

[29] V. Ya. Shkadov, Fluid Dyn. 2, 29 (1967).

[30] G. Sisoev (private communication).

[31] J. B. Keller, S. I. Rubinow, and Y. O. Tu, Phys. Fluids 16, 2052 (1973).

[32] The (normalized) cumulative integrated power equals $\left[\int_{f_{\text {low }}}^{f(i)} P(\tilde{f}) d \tilde{f}\right] /\left[\int_{f_{\text {low }}}^{f_{\text {high }}} P(\tilde{f}) d \tilde{f}\right]$, where $f_{\text {low }}$ and $f_{\text {high }}$ are lower and upper frequency bounds supporting the fundamental peak [see Fig. 12(b)], $f_{\text {low }} \leq f(i) \leq f_{\text {high, }}$, and $P(f)$ is the power at frequency $f$. 